\title{
Characterization of development, behavior and neuromuscular physiology in the phorid fly, Megaselia scalaris
}

\author{
Douglas A. Harrison, Robin L. Cooper* \\ Department of Biology, University of Kentucky, Lexington, KY 40506-0225, USA
}

Received 30 July 2002; received in revised form 24 June 2003; accepted 25 June 2003

\begin{abstract}
The Phoridae is known as 'scuttle flies' because they walk in rapid bursts of movement with short pauses between. In this study, larval locomotive behavior and development was characterized in the phorid, Megaselia scalaris. Comparison was made with the well-characterized fruit fly model, Drosophila melanogaster. Developmentally, the rate of maturation was consistently slower for Megaselia than Drosophila. This disparity was exaggerated at lower temperatures, particularly during larval development. In addition to slower growth, movements in Megaselia were also slower, as evidenced by reduced rates of larval body wall contractions and mouth hook movements. Megaselia larvae also displayed a unique behavior of swallowing air when exposed to a small pool of liquid. This permitted floating upon immersion and, therefore, might prevent drowning in the natural environment. The anatomical and physiological properties of a neuromuscular junction in the phorid larvae were also examined. The innervation of the motor nerve terminals on the ventral abdominal muscle (m6) is innervated by Type Ib and Is axons, similar to Drosophila. As in Drosophila, the Is terminals produce larger excitatory postsynaptic potentials (EPSPs) than the Ib. The amplitudes of the EPSPs in M. scalaris were reduced compared to those of D. melanogaster, but unlike D. melanogaster the EPSPs showed marked facilitation when stimulated with a $20 \mathrm{~Hz}$ train. We conclude that there may be differences in synaptic structure of the nerve terminals that could account for the different electrophysiological behaviors.
\end{abstract}

(C) 2003 Elsevier Science Inc. All rights reserved.

Keywords: Neurotransmission; Neuromuscular junction; Behavior; Development

\section{Introduction}

The genetics, development, learning, behavior and synaptic physiology of the neuromuscular junctions have been examined for decades in the fruit fly model, Drosophila melanogaster. However, other fly families show interesting characteristics that are not seen in Drosophila. Included among these are particular behaviors that may offer insights into different sensory, motor and

*Corresponding author. Tel.: +1-859-257-5950; fax: +1859-257-1717.

E-mail address: RLCOOP1@pop.uky.edu (R.L. Cooper). behavioral mechanisms and their underlying neural development and function. For example, the phorid fly, Megaselia scalaris, shows an unusual adult locomotory behavior. This species walks in short bursts with periods of rest between, hence giving phorids, the common name of 'scuttle flies' (Miller, 1979). To date, the physiological basis of this phenomenon remains to be determined.

Phorids are a diverse family in which some species have distinctive wing venation, while others may have reduced wings or no wings at all (Disney, 1994). Basic development of the common phorid species $M$. scalaris has been studied with 
regard to sex determination (Traut, 1994), oogenesis (Disney, 1994; Greenberg and Wells, 1998), fecundity (Benner, 1985) and spermatogenesis (Wolf et al., 1993). Also, descriptions of growth rates in the wild have proven useful in forensic investigations (Greenberg and Wells, 1998). However, because of the specific interest in forensic applications, these growth rates for larval $M$. scalaris have previously been determined using ground meat as a dietary source (Greenberg and Wells, 1998). Because phorids can thrive on a tremendous variety of food sources, we examined growth on a standard cornmeal-based diet commonly used for rearing Drosophila. Investigation of developmental rates showed that although, the initial egg and final adult sizes of males are very similar between the two species, the life cycle is significantly longer and the larvae and pupae are much larger for $M$. scalaris.

The characteristic locomotion of the adult $M$. scalaris has been well documented. Using highspeed video recording, it has been shown that both sexes have the distinctive scuttling behavior, though the males have a greater tendency for continuous movements (Miller, 1979). However, the mechanisms underlying this behavior and the ability to modulate this locomotive pattern by endogenous or external environmental conditions have not been determined. In contrast to the adults, phorid larvae show fluid and continuous movements that are typical of other fly species. To determine whether neuroanatomy and neurophysiology of phorid larvae are also similar to other fly species, a representative neuromuscular junction was examined. We investigated innervation profiles of the motor neurons on the larval body wall muscles ( $\mathrm{m} 6$ and $\mathrm{m} 7$ ) and characterized the synaptic properties of the neuromuscular junctions of these muscles. The $\mathrm{m} 6$ and $\mathrm{m} 7$ abdominal muscles and their associated neuromuscular junctions are the most studied muscles of larval $D$. melanogaster for neural development, target recognition, synaptic plasticity and synaptic physiology. We conclude that although, there are strong similarities in neuroanatomy of these junctions, there are several differences in the physiology of M. scalaris, including decreased excitatory postsynaptic potentials (EPSPs) and facilitation of EPSPs in response to a train of stimulatory pulses.

Lastly, in the course of behavioral analysis, we noted a unique behavior in $M$. scalaris larvae. When the phorid larvae are placed in shallow liquid, they rapidly swallow air, producing bubbles in the gut. We suggest that such behavior may have adaptational value for the species in damp environments, providing buoyancy that may allow the larvae to float in shallow water.

\section{Methods}

\subsection{Fly stock and staging of larvae}

A common wild-type laboratory strain of $D$. melanogaster, Canton S, was compared with a native strain of $M$. scalaris, which was collected on the University of Kentucky campus (Lexington, KY). Synchronous egg collections were made over $2 \mathrm{~h}$ periods on apple juice-agar plates with yeast paste. The eggs were transferred to a standard cornmeal-agar medium (Lewis, 1960) and allowed to hatch and develop with a 12:12 darklight cycle. The developmental studies were carried out at 18 and $25{ }^{\circ} \mathrm{C}$. These two temperatures were chosen as representative of the diverse conditions in which these two species thrive. Also, a substantial amount of data is known for larval development of Drosophila at these temperatures in defined conditions, which provides a means for additional comparisons ( $\mathrm{Li}$ et al., 2002). The methods used to stage fly larvae have been described previously (Campos-Ortega and Hartenstein, 1985). All the animals were reared in vials with the same cornmeal-agar-dextrose-yeast medium used to rear Drosophila (Lewis, 1960).

\subsection{Behavioral assays}

Early 3rd instar larvae were used for behavioral assays. Feeding and locomotory behavior was assessed in both species as described in Neckameyer (1996). In brief, single animals were placed on a $2 \%$ agar surface and the number of body wall contractions was counted for $2 \mathrm{~min}$, after which an animal was placed in a $2 \%$ yeast solution overlaid on an agar plate. In this condition, Drosophila larvae immediately fed, initiating a pattern of repetitive mouth hook movements. At least 10 animals were assayed in three independent experiments for the number of full mouth hook contractions in 2 min (Sewell et al., 1975). Behavioral data had previously been gathered at $25{ }^{\circ} \mathrm{C}$ for Drosophila, which allows additional comparisons to be made with M. scalaris (Neckameyer, 1996). 


\subsection{Anatomy of the neuromuscular junctions} $(N M J s)$

Morphology of the motor nerve terminals on muscle m6 was examined using fluorescentlylabeled anti-HRP viewed by confocal microscopy (Johansen et al., 1989). Fluorescent images of the nerve terminals were viewed with a Leica DM RE upright fluorescent microscope using a $40 \times$ water immersion objective. The composite images of the Z-series were collected with a Leica TCS NT/SP confocal microscope for illustration. The Leica software was used to digitize the images.

\subsection{Electrophysiology}

The preparations were taken from early and late 3rd instar larvae staged as described above. The larval dissections were performed as described in Cooper et al. (1995b). The recording arrangement and solutions are as previously described (Stewart et al., 1994; Neckameyer and Cooper, 1998). The physiological saline contained (in $\mathrm{mM}$ ): 1.0 $\mathrm{CaCl}_{2}, 70 \mathrm{NaCl}, 5 \mathrm{KCl}, 10 \mathrm{NaHCO}_{3}, 5$ trehalose, 115 sucrose, 5 BES ( $N, N$-bis[2-Hydoxyethyl]-2aminoethanesulfonic acid). All experiments were performed at room temperature $\left(19-22{ }^{\circ} \mathrm{C}\right)$. Intracellular recordings were made with the use of microelectrodes filled with $3 \mathrm{M} \mathrm{KCl}$ having a resistance of 30-60 M $\Omega$. The electrophysiological values of interest were the resting membrane potential (Rp), the EPSP amplitude for both Is and Ib motor nerve terminals in segment four of muscle six. To compare EPSP amplitudes between species, a short train of four stimuli $(20 \mathrm{~Hz})$ was given. A facilitation index (FI) was calculated by taking the ratio of the 4th EPSP amplitude over the 1st EPSP amplitude and subtracting one. Thus, if no facilitation occurred, a value of zero would be obtained. The peak amplitude values for the EPSPs were measured from the base, preceding the response, to the peak. If the responses decreased in amplitude throughout the stimulus train, a negative value would result. The mean and standard error of the mean (S.E.M.) was calculated for the resting membrane potential and EPSP responses. The individual EPSP amplitudes of the $\mathrm{Ib}$, Is as well as the mean value for the composite of recruiting $\mathrm{Ib}$ and Is together were compared between species. The input resistance of the muscle fibers was determined by plotting the slope of the current-voltage relationship based on Ohm's law for current injections (250 $\mathrm{ms}$ in duration) over the range of $\pm 5 \mathrm{nA}$.

Intracellular potentials were recorded with a 1 $\mathrm{X}$ LU head stage and an Axoclamp 2A amplifier. Field recorded excitatory postsynaptic potentials (fEPSPs) were measured with focal macropatch electrodes in conjunction with a $0.1 \mathrm{X} \mathrm{LU}$ head stage and an Axoclamp 2A amplifier in a bridge mode configuration. The fEPSP synaptic potentials were obtained using the loose patch technique by lightly placing a $10-20 \mu \mathrm{m}$ fire polished glass electrode directly over a spatially isolated varicosity along the nerve terminal that were viewed under a $40 \times$ water immersion lens (Nikon, NA $0.55)$. The macropatch electrode is specific for recordings within the region of the electrode lumen. The seal resistance was in the range of 100 $\mathrm{K} \Omega$ to $1 \mathrm{M} \Omega$. The evoked fEPSPs and field miniature excitatory postsynaptic potentials (fmEPSPs) were recorded to VHS tape and directly to MacLab Scope software (ver. 3.5.4) for calibration and measurement.

\section{Results}

\subsection{Growth}

In D. melanogaster, overall larval growth is gradual, without large punctuate jumps between instars (Alpatov, 1929; Morgan, 1926; Plunkett, 1926). This gradual growth also holds true specifically for discrete muscle groups (Li et al., 2002). Development of the $M$. scalaris larvae also shows this progressive increase in size, however, Megaselia have longer bodies as larvae and pupae as compared to identically reared Drosophila (Fig. 1). This size difference is particularly dramatic by the pupal stage (Fig. 1c). Interestingly, the eggs of Megaselia are only slightly larger than $D$. melanogaster, with distinctly different morphology of the chorion (Fig. 1a). Furthermore, the adult males of the species are also very similar in size. Therefore, despite great disparity in sizes of the intermediate stage animals, the beginning embryos and resulting adult males are quite similar in $D$. melanogaster and M. scalaris. In contrast, the phorid females are much larger than either the males or the D. melanogaster females. Although D. melanogaster females are larger than males, this sexual dimorphism is much more pronounced in M. scalaris. It should be noted that M. scalaris is a scavenger species and can grow in the wild 

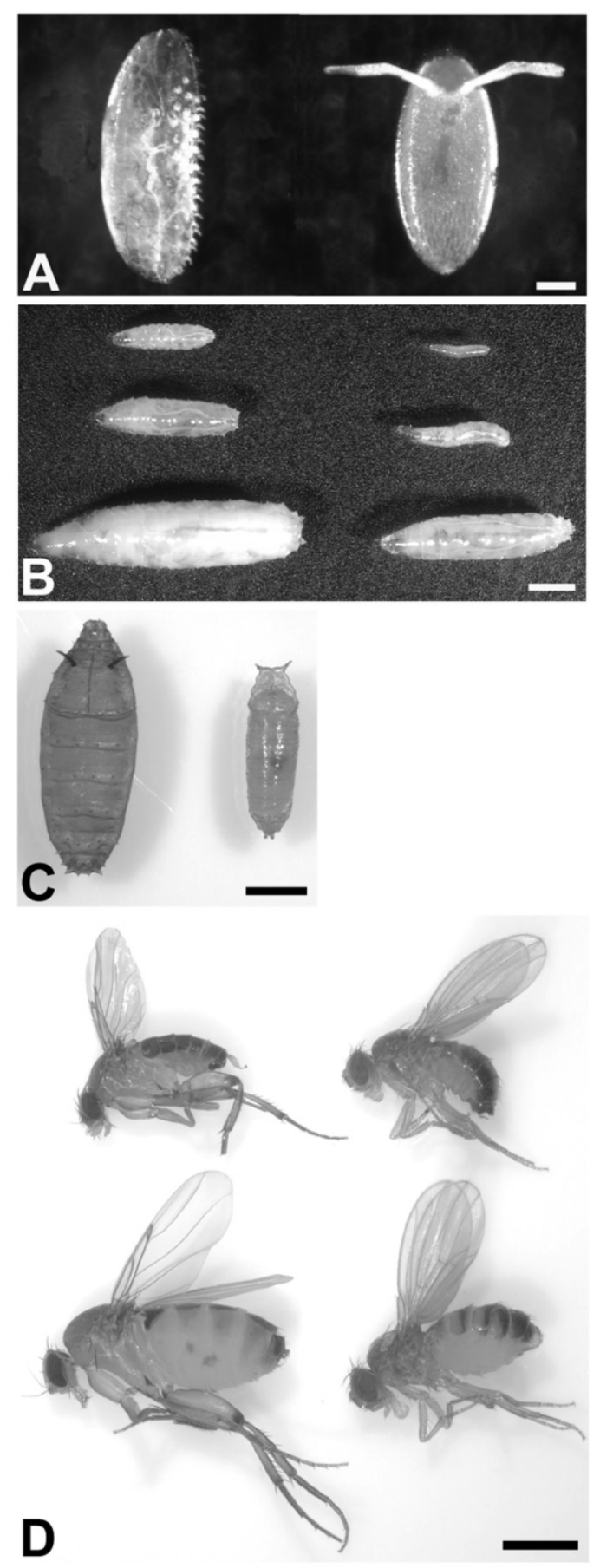

Fig. 1. Comparison of morphology and size of M. scalaris and D. melanogaster throughout development. Though the eggs of the two flies are similar in size, (a) all larval instars, (b) pupae, (c) and adult females, (d bottom row) of M. scalaris are significantly larger than D. melanogaster. M. scalaris is on the left side of each panel. The adult males of both species are shown in the upper row of $\mathrm{d}$. Scale bar is $0.1 \mathrm{~mm}$ for panel A and $1 \mathrm{~m}$ for $\mathrm{B}, \mathrm{C}$ and $\mathrm{D}$. on a variety of media, including rotting meat. In the laboratory, this species is also reared on various food sources, but is frequently grown on a modified Drosophila medium consisting of a cornmealbased food supplemented with a small piece of meat (Willhoeft and Traut, 1990). However, for the purposes of this study, both species were reared on an identical diet, comprised of a standard cornmeal-agar Drosophila medium without any meat.

Consistent with their greater size, the phorids developed more slowly than Drosophila. Comparison was made in the developmental rate through embryonic hatching and through to pupation for $M$. scalaris and D. melanogaster at both 18 (Fig. $2 \mathrm{a}_{1}$ and $\mathrm{b}_{1}$ ) and $25{ }^{\circ} \mathrm{C}$ (Fig. $2 \mathrm{a}_{2}$ and $\mathrm{b}_{2}$ ). Both embryonic and larval growth is approximately 1.5 times longer in the phorids than in Drosophila reared at $25{ }^{\circ} \mathrm{C}$ (Fig. 2c). The relative rate also holds for embryonic development of the two species at $18{ }^{\circ} \mathrm{C}$. However, larval development is dramatically slower (three-fold) for phorids than for Drosophila at $18{ }^{\circ} \mathrm{C}$ (Fig. $2 \mathrm{~b}_{1}$ ). The extended larval stages of $M$. scalaris as compared with $D$. melanogaster likely reflect the substantially greater amount of growth required in the phorids to reach pupariation. Both species tend to pupariate out of the food in a dry location, such as on the lid or walls of the growth chambers.

\subsection{General behaviors}

Since the adult locomotive behavior is so strikingly different between these two species, we predicted that there may be some difference in larval movement. To test this prediction, two standard behavioral assays of body movements were used: the rate of body wall contraction and the rate of mouth hook movements (Neckameyer, 1996). Animals in the early wandering 3rd instar were used for both species. In contrast to adult locomotion, the movements of phorid larvae are fluid and continuous. Yet, in comparison with Drosophila larvae, the locomotor behavior of the body wall contractions was substantially reduced for M. scalaris at $18{ }^{\circ} \mathrm{C}$ (Fig. $3 \mathrm{a}_{1} ; P<0.05, t$-test, $n=20$ ), though not significantly different at $25^{\circ} \mathrm{C}$ (Fig. $3 \mathrm{a}_{2}$ ). Even more notable differences were observed in the number of mouth hook movements when the larvae were exposed to a $2 \%$ yeast solution overlaid on an agar plate. M. scalaris are less active in eating the food, and thus they have 

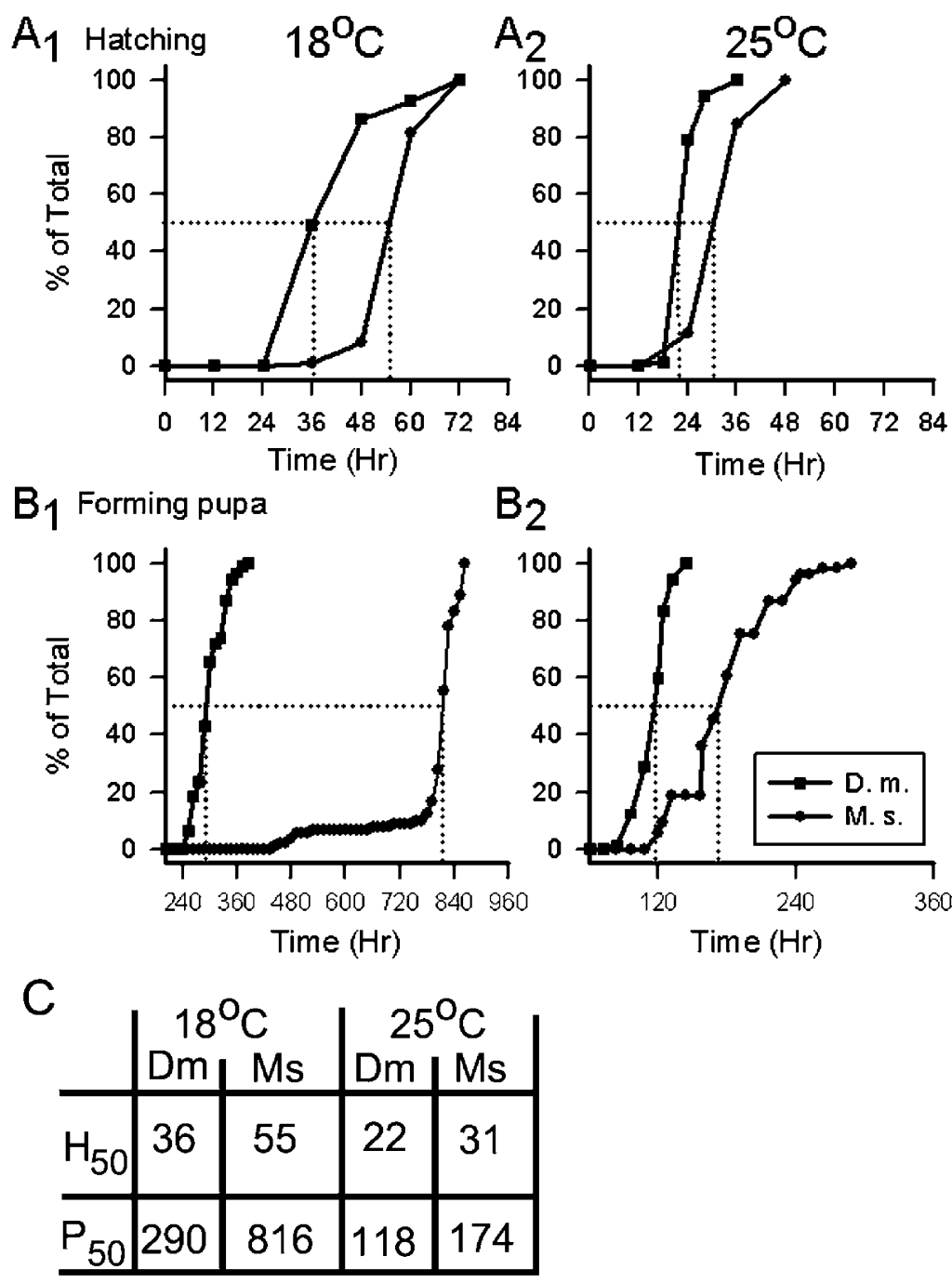

Fig. 2. A rank sum of the number of embryos hatched and animals pupated over time for both M. scalaris and D. melanogaster. M. scalaris have longer embryonic $\left(\mathrm{a}_{1}\right)$ and dramatically longer larval developmental periods at $18{ }^{\circ} \mathrm{C}\left(\mathrm{b}_{1}\right)$. The differences between the two species are not as dramatic at $25{ }^{\circ} \mathrm{C}\left(\mathrm{a}_{2}\right.$ and $\left.\mathrm{b}_{2}\right)$. The time required for $50 \%$ of the embryos to develop from egg deposition to hatching $\left(\mathrm{H}_{50}\right)$ and for $50 \%$ of the larvae to develop from egg deposition to pupariation $\left(\mathrm{P}_{50}\right)$ is indicated for each species at each temperature (c).

fewer mouth hook movements than $D$. melanogaster $\left(P<0.05, t\right.$-test, $n=20$; Fig. $3 b_{1}$ and $\left.b_{2}\right)$. The differences are greater at $25{ }^{\circ} \mathrm{C}$ than at $18{ }^{\circ} \mathrm{C}$. The reduced feeding rate in $M$. scalaris is because larvae expended effort in swallowing air when placed on a yeast solution that was nearly able to immerse the animal. D. melanogaster did not elicit this type of behavior, yet both species were able to breathe under these conditions by raising the spiracles out of the solution to maintain access to air. This air swallowing in M. scalaris caused the gut to fill with air bubbles (Fig. 4). Because this behavior was only observed upon exposure to a liquid environment, we hypothesized that this might be a survival strategy to allow the animal to float if immersed. To test this idea, we exposed both $M$. scalaris and D. melanogaster to the $2 \%$ yeast solution used for the behavioral assay for 10,20 or $60 \mathrm{~min}$. This afforded the larvae an opportunity to swallow air. Afterwards, we placed the larvae into a $100 \mathrm{ml}$ beaker containing room temperature water $(80 \mathrm{ml})$. All of the D. melanogaster $(n=60)$ sank to the bottom and died after a short time, whereas many of the M. scalaris 

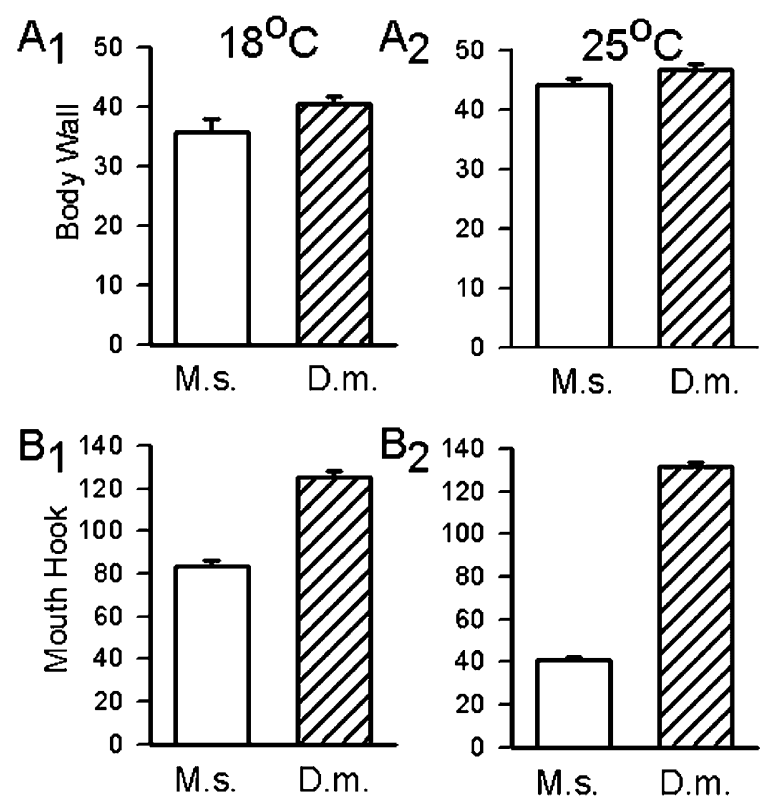

Fig. 3. The locomotor behavior assessed by the number of body wall contractions per minute (averaged over $2 \mathrm{~min}$ ) for early 3rd instar is reduced for M. scalaris as compared to D. melanogaster at $18{ }^{\circ} \mathrm{C}\left(\mathrm{a}_{1}\right)$ but not at $25{ }^{\circ} \mathrm{C}\left(\mathrm{a}_{2}\right)$. The number of mouth hook movements per minute (averaged over $2 \mathrm{~min}$ ) when exposed to a $2 \%$ yeast solution was substantially lower for M. scalaris than D. melanogaster at both temperatures (b).

larvae fared much better. After only a 10 min preexposure to yeast paste, eight out of 10 larvae sank rapidly, while the remaining two were unable to float and bobbed within the water column. After a 20 min pre-exposure, 11 out of 20 larvae floated (10 entirely on the surface and one within the water column), and even after $30 \mathrm{~min}$ in the water, only $50 \%$ sank and died. When given a $60 \mathrm{~min}$ pre-exposure in yeast paste, nine out of 10 larvae floated and survived for $30 \mathrm{~min}$ in water. When a flat wooden ramp (i.e. a clinical type of tongue depressor) is placed in the beakers, the floating larvae of M. scalaris crawled up on the wood and were able to fully escape the water. We conclude that the air swallowing response can be a successful means of preventing drowning, depending upon the amount of time permitted before complete immersion.

\subsection{Anatomy of larval muscle and NMJs}

The innervation pattern for the neuromuscular junctions on muscle six (m6) and muscle seven (m7) within abdominal segment four is different in late 3rd instar M. scalaris than for D. melanogaster. In Drosophila, the terminals are primarily Type I endings from the two major axons (Is and Ib) (Atwood et al., 1993). The two neurons innervate $\mathrm{m} 6$ and $\mathrm{m} 7$ in the region, where the two muscles are in close apposition to one another. The terminals of the axons branch from this apposition point on to the muscle (Fig. 5a). In $M$. scalaris, a nerve branch innervating $\mathrm{m} 6$ frequently arises from a lateral edge of the muscle (Fig. 5b, double arrow). In addition, it is unusual to observe in D. melanogaster such separation in the two

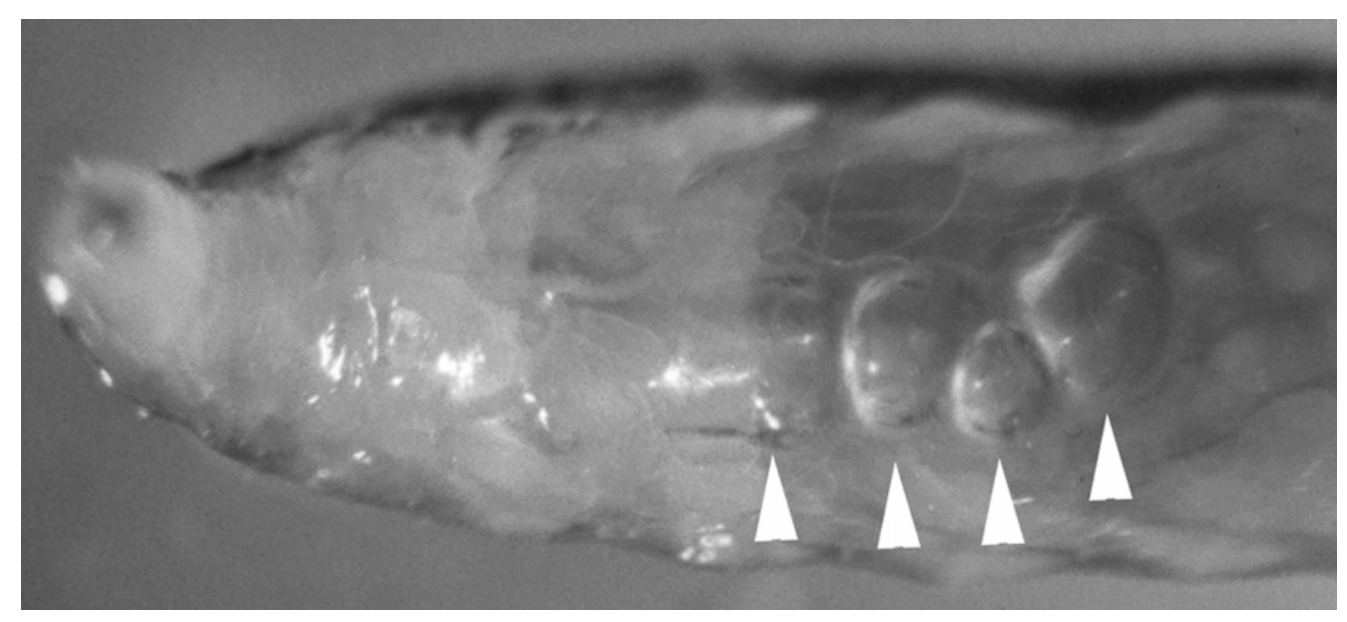

Fig. 4. When partly immersed in aqueous solution, M. scalaris swallowed air, filling the gut with bubbles (arrowheads) that aided larvae in floating (see text). D. melanogaster did not show this behavior. 

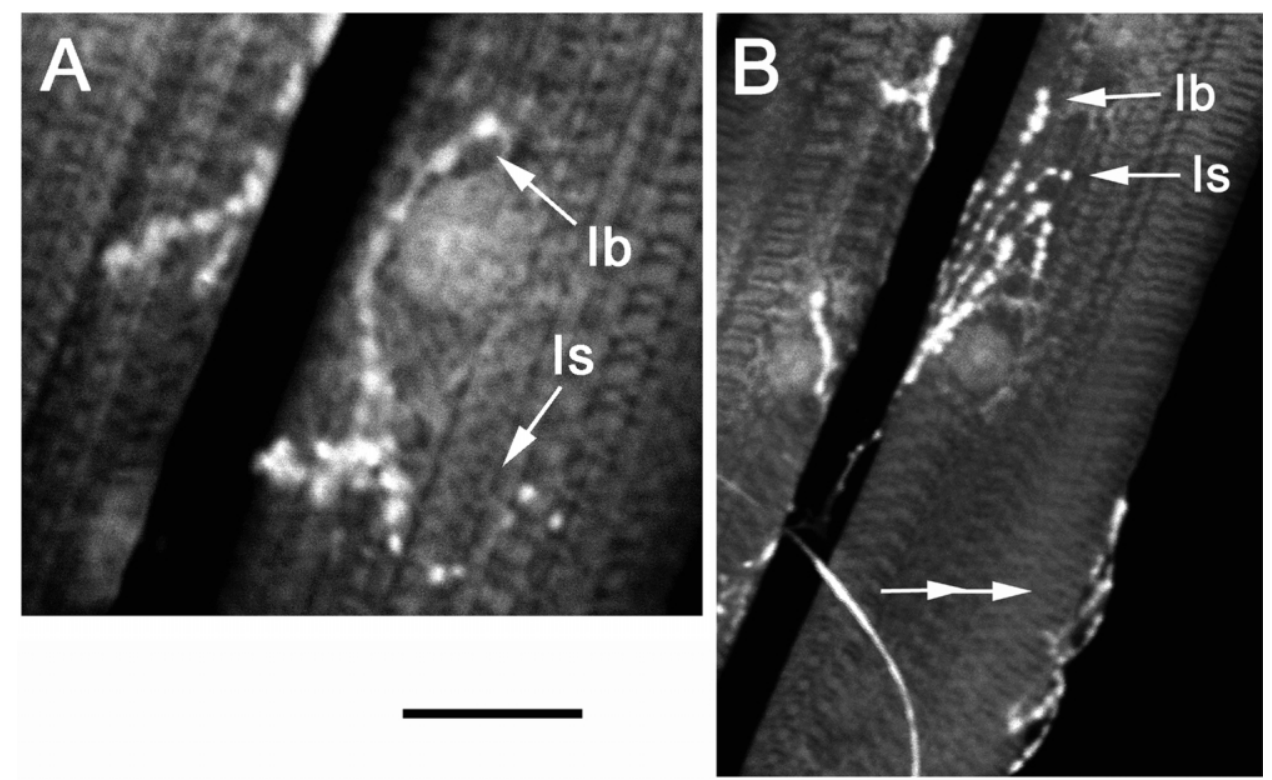

Fig. 5. Neuromuscular junctions on abdominal muscles six and seven within abdominal segment four at the late 3rd instar for $M$. scalaris. The larvae were raised at $18{ }^{\circ} \mathrm{C}$. Motor terminals were labeled with fluorescein-tagged anti-HRP and imaged with a confocal microscope. Muscle six is the muscle on the right side of both panels. Note the lateral innervation on m6 in panel B (double arrowhead). The Ib and Is terminals are readily identifiable with the Ib terminals containing bigger varicosities as compared to the smaller ones for the Is terminal. Scale bar: $34 \mu \mathrm{m}$ for A and $51 \mu \mathrm{m}$ for B.

nerve terminals as seen often in $M$. scalaris (Fig. 5 b). As for D. melanogaster, the anatomic profiles of the terminals match the Is and Ib terminal classification for M. scalaris. The type Ib (big) terminals have large varicosities as compared to the Type Is (small) terminals with smaller varicosities.

\subsection{Synaptic physiology at the NMJ}

We assessed the synaptic transmission of the neuromuscular junctions of $M$. scalaris in comparison to D. melanogaster for two well established motor neurons. The terminals of the $\mathrm{Ib}$ and Is motor neurons are well known in structure and function in D. melanogaster and for a species of flesh fly, Sarcophaga bullata (Feeney et al., 1998), so we chose to investigate these two motor nerves on muscle six within the 4th abdominal segment in M. scalaris for comparative physiological purposes. The Ib axon produces smaller EPSPs than the Is axon in both $M$. scalaris and D. melanogaster. Fig. 6a shows representative examples of the EPSPs of a larva in the late 3rd instar. To compare differences and variability between species, the mean and S.E.M. for the composite EPSPs (Ib+ Is) are shown (Fig. 6c). The amplitudes of the composite responses were significantly lower in M. scalaris as compared to D. melanogaster $(P<$ $0.05, t$-test, $n=13)$. The individual responses of the $\mathrm{Ib}(5.6 \mathrm{mV}$ mean \pm 0.5 S.E.M. $)$ and Is $(11.2$ $\mathrm{mV}$ mean \pm 0.9 S.E.M.) responses were also lower in M. scalaris as compared to the $\mathrm{Ib}(14.6 \mathrm{mV}$ mean \pm 0.8 S.E.M. $)$ and Is $(25.8 \mathrm{mV}$ mean \pm 1.3 S.E.M. $)$ in D. melanogaster $(P<0.05, n=13)$. The same physiological saline (HL3) was used to examine physiological profiles in both species. The saline was developed for $D$. melanogaster but also appeared sufficient for $M$. scalaris. The average values for the resting membrane potentials were less negative in $M$. scalaris as compared to D. melanogaster (Fig. 6d). Additional characterization of the synaptic efficacy was carried out in the presence of short stimulus trains. Trains of four pulses delivered at $20 \mathrm{~Hz}$ are a commonly used paradigm in physiologic studies of $D$. melanogaster. With this stimulation paradigm in M. scalaris, an unexpected result occurred. The amplitude of the EPSPs within the response train facilitated (Fig. 6b). In D. melanogaster, the EPSP responses 
(a)

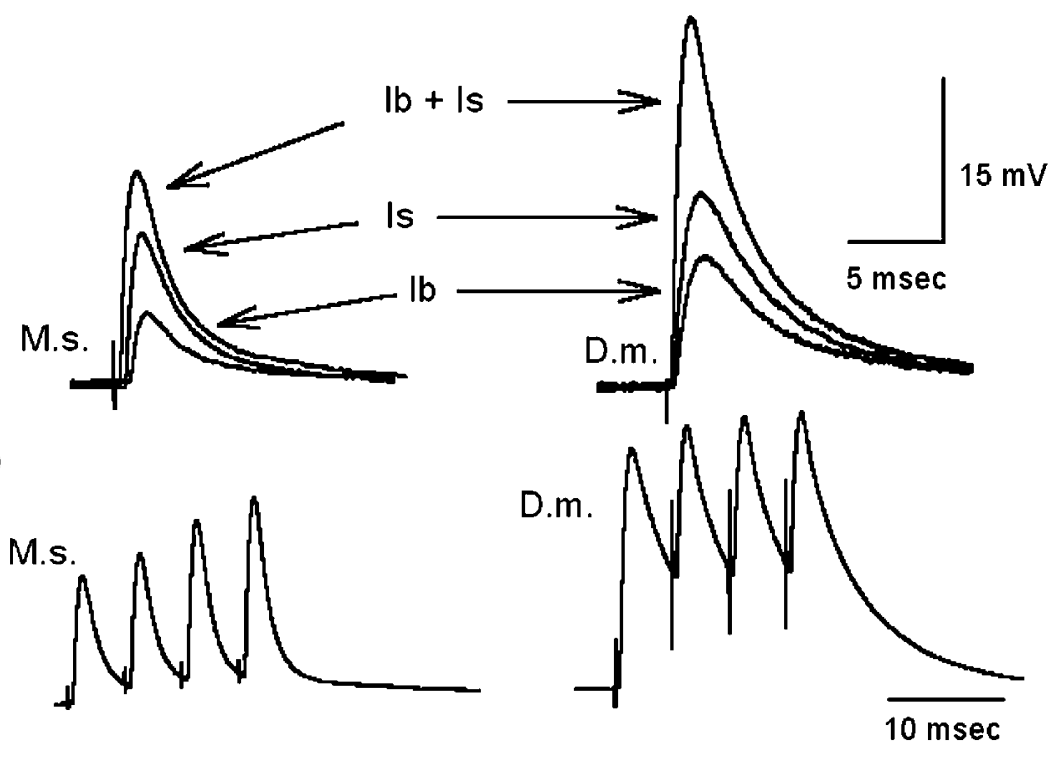

(c)

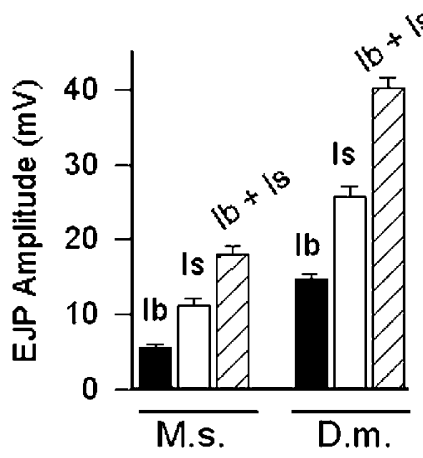

(d)

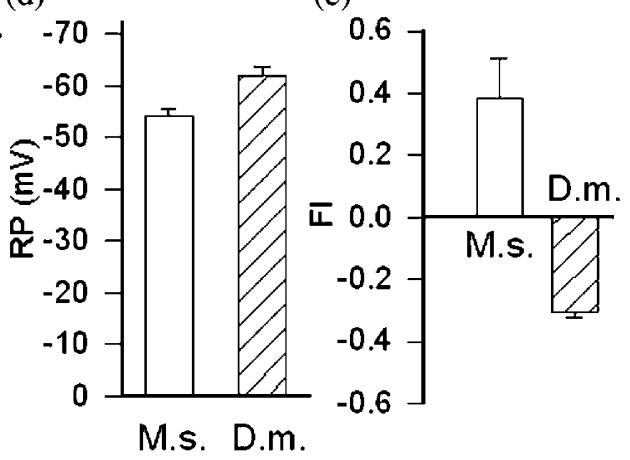

Fig. 6. In abdominal muscle six, the Ib axon produces a smaller EPSPs than the Is axon for both species. Representative examples are shown (a) of EPSPs recorded within the late 3rd instar while either the Ib or Is axons were selectively stimulated. Short stimulus trains of four pulses delivered at $20 \mathrm{~Hz}$ indicated that the responses facilitated for M. scalaris as compared to D. melanogaster (b). The average responses for the $\mathrm{Ib}$ and Is as well as the composite $\mathrm{Ib}+\mathrm{Is}$ EPSP for both species are shown (c) along with the average resting membrane potential (d). The facilitation index (FI) shows that in the same physiological environment, synaptic transmission is different between M. scalaris and D. melanogaster with Megaselia having more pronounced facilitation (e). Error bars are for standard error of the mean. All recordings were made in HL3 saline (see Section 2). The scale bar in a is for both M. scalaris (M.s.) and D. melanogaster (D.m.). The same time scale in $\mathrm{b}$ is also for both species.

depress in size, thus producing a negative facilitation index (Fig. 6b). The facilitation index for the two species is shown in Fig. 6e. In all the measures indicated, there is a significant difference $(P<0.05)$ between the two species.

To determine if a difference in the muscle fiber input resistance might contribute to the differences of the EPSP amplitudes between the species, measures were taken in the same conditions for both species. The input resistance for $M$. scalaris was on average $7.6 \mathrm{M} \Omega( \pm 1.1 \mathrm{M} \Omega$, S.E.M.; $n=6)$ and for $D$. melanogaster, the input resistance under the same conditions has been reported to be on average $7.5 \mathrm{M} \Omega( \pm 0.3 \mathrm{M} \Omega$, S.E.M.; $n=6$; Stewart et al., 1996). Thus, there is no significant difference in muscle fiber input resistance between the two species.

To quantify the field recorded excitatory postsynaptic potentials (fEPSPs), focal macropatch recordings were made over selective regions of the $\mathrm{Ib}$ and Is nerve terminals. To insure that the fEPSPs and EPSPs corresponded with the selective stimulation, the Ib or Is axons, EPSPs were recorded in muscle seven $(\mathrm{m} 7)$, while fEPSPs were recording 

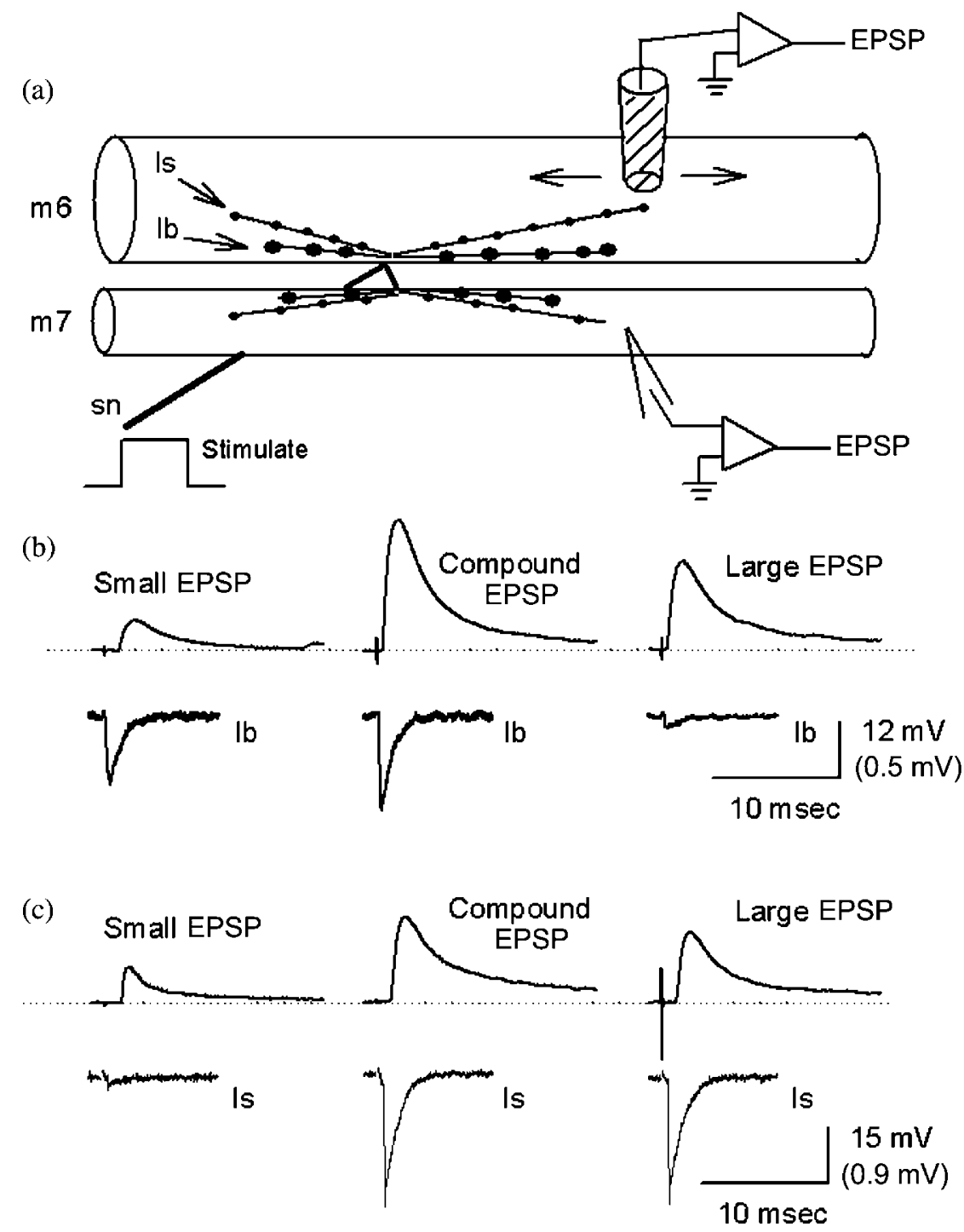

Fig. 7. In order to match synaptic transmission with terminal types, simultaneous fEPSPs and intracellular obtained EPSPs recordings are made while recruiting selectively the axons of the segmental nerve $(\mathrm{sn})$. The EPSPs were monitored in m7, while the fEPSPs were monitored over the two terminal strings of small (Is) or large (Ib) varicosities on m6 (a). The fEPSPs obtained from small varicosities were only observed when the small EPSP or compound EPSP was recorded, but not the single large EPSP (b). In contrast, the fEPSPs obtained from large varicosities were only observed when the large EPSP or compound EPSP was recorded, but not the small large EPSP (c). Scale bars are in $\mathrm{mV}$ for the EPSPs and the fEPSPs (in parentheses).

from selective varicosities of the two types of terminals on $\mathrm{m} 6$. The axons of $\mathrm{Ib}$ and Is bifurcate to innervate both $\mathrm{m} 6$ and $\mathrm{m} 7$ muscles within each hemi-segment. This stimulation and selective recording procedure was similar to that described by Kurdyak et al. (1994) in differentiating the responses from Ib and Is terminals in Drosophila except that we recorded EPSPs in m6. The modified procedure allowed more varicosities to be sampled on $\mathrm{m} 6$ without producing damage at the site of recordings (Fig. 7). We consistently observed that the Is terminals produced larger fEPSPs with more multiples of quantal content than the Ib terminals (Fig. 8a and b). This is observed for both species and has previously been well documented in D. melanogaster (Kurdyak et al., 1994; Stewart et al., 1994; Cooper et al., 1995b). The field potentials recorded directly over 
(a)
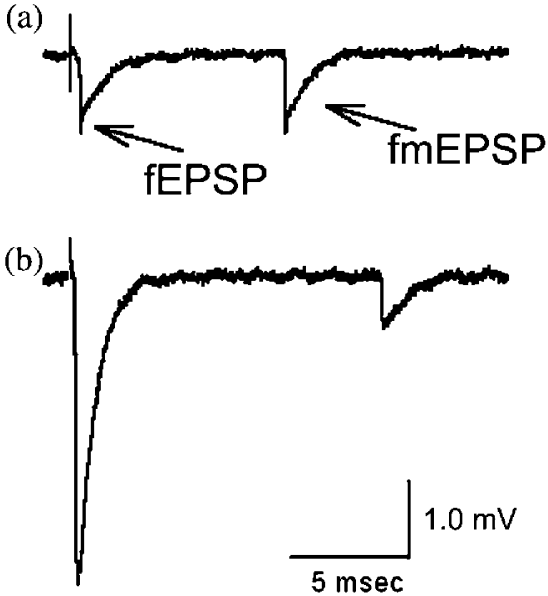

(c)

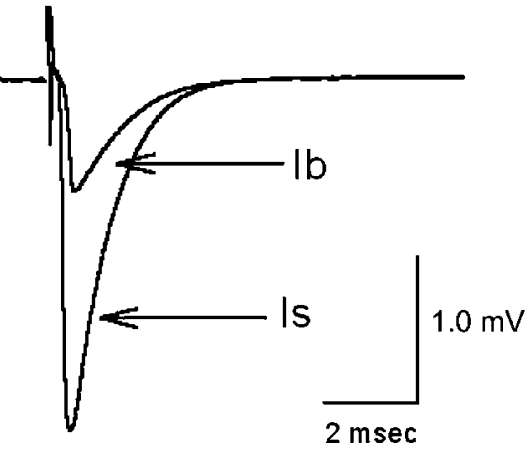

Fig. 8. To quantify the field excitatory postsynaptic potentials (fEPSPs), focal macropatch recordings were made over visualized varicosities on the nerve terminals. The Ib terminals (a) produces smaller fEPSPs with fewer multiples of quantal content than the Ib terminals (b). This is observed for both species and has been well documented in D. melanogaster (Kurdyak et al., 1994; Stewart et al., 1994; Cooper et al., 1995b). The stimulus artifact is followed by the evoked fEPSP and in the some instances, a spontaneous quantal event or field miniature EPSP (fmEPSP) is recorded. An ensemble average of 400 evoked responses while recording from a single $\mathrm{Ib}$ or a Is varicosity is shown. The Is varicosities on average have a greater quantal content per evoked event (c). The scales are the same for $a$ and $b$.

the Ib varicosities would depict release of only single quanta or failures when evoked for $M$. scalaris. This is not a common occurrence for $\mathrm{Ib}$ terminals in D. melanogaster, since multiple quantal are revealed during an evoked stimulus and rarely are failures in release observed. The single quantal size is observed by recording spontaneous or field miniature recorded excitatory postsynaptic potentials (fmEPSPs) as seen later in the traces for both the $\mathrm{Ib}$ and Is responses (Fig. 8a and b). Averages of 400 evoked trials depict the overall difference in synaptic efficacy for the varicosities on the Ib and Is terminals. The average of evoked fEPSPs for the $\mathrm{Ib}$ is markedly smaller than that for the Is terminals (Fig. 8c).

\section{Discussion}

Although, there are distinct morphological differences between M. scalaris and D. melanogaster, both develop from eggs of very similar size that grow to be adults that are comparable in size, at least for males (M. scalaris adult females are substantially larger than males or D. melanogaster of either sex). Yet along the way, the larvae and pupae of Megaselia are much larger than comparably staged Drosophila. Consistent with the larger larval and pupal sizes, the larval development of M. scalaris is strikingly slower at $18{ }^{\circ} \mathrm{C}$ and somewhat slower at $25{ }^{\circ} \mathrm{C}$ than that of D. melanogaster under the same environmental conditions. The exaggerated disparity between the species at $18{ }^{\circ} \mathrm{C}$ may indicate that $M$. scalaris is more stressed at that lower temperature than $D$. melanogaster.

In an earlier study (Greenberg and Wells, 1998), in which M. scalaris was raised on ground beef, the average minimum times to pupariation at various temperatures were approximately $100 \mathrm{~h}$ $\left(29{ }^{\circ} \mathrm{C}\right), 155 \mathrm{~h}\left(22{ }^{\circ} \mathrm{C}\right)$ and $200 \mathrm{~h}\left(19{ }^{\circ} \mathrm{C}\right)$. In our study, pupariation started approximately $250 \mathrm{~h}$ at $25{ }^{\circ} \mathrm{C}$ and $420 \mathrm{~h}$ at $18{ }^{\circ} \mathrm{C}$, when larvae were maintained on a cornmeal diet. Though it is impossible to make quantitative comparisons between these separate experiments, partly because the rearing temperatures used were different, we can make estimates of the growth by extrapolation from these data. Based on the times to pupariation at 29 and $22{ }^{\circ} \mathrm{C}$, we know that the time to pupariation at $25{ }^{\circ} \mathrm{C}$ when reared on ground meat must be between 100 and $155 \mathrm{~h}$, and most likely would be approximately $130 \mathrm{~h}$. When compared with the $250 \mathrm{~h}$ required to reach pupariation on a cornmeal diet, we must conclude that $M$. scalaris grows substantially faster on meat than the vegetable-based food used in this study. Thus, in spite of the capability of this phorid to survive on a wide variety of media, there is a clear difference in the quality of these two types to support optimal growth of $M$. scalaris. However, this does not indicate that $M$. scalaris grows poorly on the cornmeal medium. Although, they develop more slowly than animals raised on meat, even the 
cornmeal-based food supports robust proliferation of these phorids, producing large numbers of viable progeny.

Significant differences in specific behaviors of $M$. scalaris and D. melanogaster were also noted. The rates of body wall contractions and mouth hook movements are both slower in M. scalaris. However, the substantially slower mouth hook movements in M. scalaris are due, at least in part, to replacement of feeding activities with a novel and unexpected behavior: larvae swallow air when exposed to an aqueous environment. This behavior permits the larvae to float, even in deep pools of liquid, if permitted sufficient time to gulp air prior to being exposed to deeper water. This would appear to be a successful survival strategy in the event that larvae become immersed in liquid in their environment. This might be particularly effective in situations where a larva may be trapped in a gradually increasing volume of liquid, such as during a rainstorm. However, based on our tests using only brief exposure prior to total immersion, sudden complete immersion of the larva would likely not permit sufficient swallowing of air to build buoyancy before sinking. Thus, the survival utility of this behavior is limited only to situations in which immersion would be gradual. As for body wall contractions between the two examined species, no significant difference was obtained between the two temperatures tested. Alteration in temperature has substantial effects on synaptic transmission at NMJs for insects. Such slowing down of synaptic transmission is likely to account for the slightly slower movements in body wall contractions and reduction in mouth hook movements at the lower temperatures, since they are not able to maintain the same degree of synaptic transmission.

In addition to differences in growth rate, size and some behaviors, the architecture of the neuromuscular junction and electrophysiology of the larvae are also different. The $\mathrm{Ib}$ and Is motor nerve terminals and the corresponding muscle six (m6) in segment four of the 3rd instar M. scalaris larvae show differences in their properties as compared to $D$. melanogaster. The motor nerve terminal innervation on muscle six is erratic in $M$. scalaris as compared to the more consistent patterns observed in D. melanogaster (Li et al., 2002). The physiological differences were that the EPSPs for both the $\mathrm{Ib}$ and Is terminals are smaller and the resting membrane potential reduced (less negative) in $M$. scalaris. The time constants of the rise and decay are similar. However, the EPSPs show facilitation in M. scalaris, whereas in D. melanogaster the responses depress in the same physiological saline. Similarities exist between the two species in that they both have Type Ib and Is terminals innervating the $\mathrm{m} 6$ and $\mathrm{m} 7$ abdominal muscles. Also, the Is terminals in both species produce a larger EPSP than do the Ib terminals, and the field potentials for the Is varicosities are larger than those for the varicosities of the Ib. These differences are likely due to the increased number of vesicular events associated with the synapses contained in the Is terminal as compared to the $\mathrm{Ib}$ terminal.

Since the m6 muscle is substantially larger in M. scalaris in the wandering 3 rd instar as compared to D. melanogaster, one may expect the amount of innervation on the muscle from the motor terminals to be proportional to the surface area or volume. We found it difficult to quantify the length of the $\mathrm{Ib}$ and Is motor nerve terminals of $M$. scalaris as compared D. melanogaster, since innervation occurred on the ventral and lateral sides on the m6 muscle as compared to the dorsal surface innervation in D. melanogaster. In growth studies of $D$. melanogaster from 1 st instar to late 3rd instar stages, both $\mathrm{m} 6$ and its motor axons $\mathrm{Ib}$ and Is were characterized by second order regressions after the data was transformed with a natural $\log$. The number of varicosities and terminal length of Is axon increases more rapidly than for the $\mathrm{Ib}$ axon throughout development (Li et al., 2002). One might expect a reduced growth rate for $M$. scalaris, since their larval develop is reduced at $18{ }^{\circ} \mathrm{C}$ as compared to D. melanogaster.

The reduced amplitude of the EPSPs for the $\mathrm{Ib}$ and Is in M. scalaris might be a result of less innervation, but there could also be biophysical differences in the postsynaptic muscle fiber to account for this, since the events are measured in the muscle. However, the time constants for the rise and decay of the compound EPSPs were not significantly different between the two species (data not shown). Since there is no significant difference in the input resistance of the muscle fibers of the two species, which could have impacted the rising phase of the EPSPs, one would then predict that less transmitter is released from each synaptic varicosity or the receptivity of the muscle fiber is decreased in M. scalaris. Synaptic field potential recordings obtained directly over discrete- 
ly isolated varicosities of the $\mathrm{Ib}$ and Is terminals did reveal smaller evoked field potential for both terminals in M. scalaris. This is likely due to the fact that the mean quantal content for varicosities of the Ib and Is terminals for M. scalaris is less than that previously reported for $D$. melanogaster (Cooper et al., 1995b; Stewart et al., 1996; Kurdyak et al., 1994).

The homoeostatic regulation of synaptic efficacy throughout development in D. melanogaster is not completely elucidated, but many studies note a strong correlation between the length of motor nerve terminals and synaptic strength of the varicosities with development of the muscle. Several studies have used genetic manipulations to address these issues in D. melanogaster (Petersen et al., 1997; Davis and Goodman, 1998) and mice (Sandrock et al., 1997). Much of the structural regulation is at the level of the synapse. It has been demonstrated that homoeostatic regulation in nerve-muscle matching is correlative to the complexity of the synapses in containing greater or fewer active zones (Stewart et al., 1996). Active zones are structural entities on the presynaptic membrane where vesicles dock to fuse with the membrane. Such differences in synaptic complexity account for the differences between the Ib and Is varicosities in D. melanogaster (Atwood et al., 1993; Kurdyak et al., 1994) and for high and low output synapses in crustaceans (Atwood and Cooper, 1995, 1996a,b; Cooper et al., 1995a, 1996a,b; Govind and Chiang, 1979; King et al., 1996). To resolve this issue in relation to synaptic efficacy in M. scalaris, electron micrographic studies of the motor nerve terminals will need to be undertaken. To obtain an accurate assessment of the synaptic area and numbers of active zones per synapse, one would need serial, cross-sections and reconstruction via electron micrographs. At this point in time, we were not able to conduct such studies.

Future comparative investigations with other flies regarding the air gulping behavior in $M$. scalaris would be of interest to determine how broadly throughout insect evolution this potentially advantageous behavior is employed. In addition, further investigation into the composition of the larval hemolymph is needed in M. scalaris, since the synaptic transmission is quite different (i.e. low EPSP amplitude and high facilitation) from that of $D$. melanogaster. This could be accounted for, in part, by a low $\mathrm{Ca}_{2}{ }^{+} / \mathrm{Mg}^{+}$ratio in the HL3 media for $M$. scalaris. The difference we report may be due to non-physiologic based ionic content for this species. Slight alterations in ionic composition of the designed hemolymph-like salines for D. melanogaster show substantial changes in the characteristics of the EPSPs (Ball et al., 2003; Stewart et al., 1994). Lastly, it would also be beneficial to obtain comparative information on the synaptic structure of the $\mathrm{Ib}$ and Is terminals.

\section{Acknowledgments}

We thank Mr Ryan Ball (University of KY) and Ms Racennah Braxton (Talladega college, AL) for helping to conduct behavioral assays used in this study. Dr Susan Harrison assisted with photography. Funding was provided by NSF grants IBN0091535 (DH), IBN-9808631 (RLC) and NSF-ILI-DUE 9850907 (RLC). We thank $\mathrm{Mr}$ Mike Sharkey (Department of Entomology, University of Kentucky) and Dr Brian Brown (Associate Curator of Entomology, Natural History Museum of Los Angeles County, CA) for species identification.

\section{References}

Alpatov, W.W., 1929. Growth and variation of the larvae of Drosophila melanogaster. J. Exp. Zool. 52, 407-437.

Atwood, H.L., Cooper, R.L., 1995. Functional and structural parallels in crustaceans and Drosophila neuromuscular systems. Am. Zool. 35, 556-565.

Atwood, H.L., Cooper, R.L., 1996a. Assessing ultrastructure of crustacean and insect neuromuscular junctions. J. Neurosci. Methods 69, 51-58.

Atwood, H.L., Cooper, R.L., 1996b. Synaptic diversity and differentiation: crustacean neuromuscular junctions. Invert. Neurosci. 1, 291-307.

Atwood, H.L., Govind, C.K., Wu, C.-F., 1993. Differential ultrastructure of synaptic terminals on ventral longitudinal abdominal muscles in Drosophila larvae. J. Neurobiol. 24, 1008-1024.

Ball, R., Xing, B., Bonner, P., Shearer, J., Cooper, R.L., 2003. Long-term maintenance of neuromuscular junction activity in cultured Drosophila larvae. Comp. Biochem. Physiol. A 134, 247-255.

Benner, D.B., 1985. Oocyte development and fecundity in Megaselia scalaris (Phoridae: Diptera). Int. J. Entomol. (Honolulu) 27, 280-288.

Campos-Ortega, J.A., Hartenstein, V., 1985. The embryonic development of Drosophila melanogaster. Springer-Verlag, Berlin.

Cooper, R.L., Marin, L., Atwood, H.L., 1995a. Synaptic differentiation of a single motor neuron: conjoint definition of transmitter release, presynaptic calcium signals, and ultrastructure. J. Neurosci. 15, 4209-4222.

Cooper, R.L., Stewart, B.A., Wojtowicz, J.M., Wang, S., Atwood, H.L., 1995b. Quantal measurement and analysis 
methods compared for crayfish and Drosophila neuromuscular junctions and rat hippocampus. J. Neurosci. Methods 61, 67-78.

Cooper, R.L., Harrington, C., Marin, L., Atwood, H.L., 1996a. Quantal release at visualized terminals of crayfish motor axon: intraterminal and regional differences. J. Comp. Neurol. 375, 583-600.

Cooper, R.L., Winslow, J., Govind, C.K., Atwood, H.L., 1996b. Synaptic structural complexity as a factor enhancing probability of calcium-mediated transmitter release. J. Neurophysiol. 75, 2451-2466.

Davis, G.W., Goodman, C.S., 1998. Synapse-specific control of synaptic efficacy at the terminals of a single neuron. Nature. 392, 82-86.

Disney, R.H.L., 1994. Scuttle Flies: The Phoridae. Chapman and Hall, London, Glasgow.

Feeney, C.J., Karunanithi, S., Pearce, J., Govind, C.K., Atwood, H.L., 1998. Motor nerve terminals on abdominal muscles in larval flesh flies, Sarcophaga Bullata: comparison with Drosophila. J. Comp. Neurobiol. 402, 197-209.

Govind, C.K., Chiang, R.G., 1979. Correlation between presynaptic dense bodies and transmitter output at lobster neuromuscular terminals by serial section electron microscopy. Brain Res. 161, 377-388.

Greenberg, B., Wells, J.D., 1998. Forensic use of Megaselia abdita and M. scalaris (Phoridae: Diptera): case studies, development rates, and egg structure. J. Med. Entomol. 35, 205-209.

Johansen, J., Halpern, M.E., Johansen, K.M., Keshishian, H., 1989. Stereotypic morphology of glutamatergic synapses on identified muscle cells of Drosophila larvae. J. Neurosci. 9, 710-725.

King, M.J.R., Atwood, H.L., Govind, C.K., 1996. Structural features of crayfish phasic and tonic neuromuscular junctions. J. Comp. Neurol. 372, 618-626.

Kurdyak, P., Atwood, H.L., Stewart, B.A., Wu, C.-F., 1994. Differential physiology and morphology of motor axons to ventral longitudinal muscle in larval Drosophila. J. Comp. Neurol. 350, 463-472.

Lewis, E.B., 1960. A new standard food medium. Drosoph. Inf. Serv. 34, 117-118.

Li, H., Peng, X., Cooper, R.L., 2002. Development of Drosophila larval neuromuscular junctions: maintaining synaptic strength. Neuroscience 115, 505-513.
Miller, P.L., 1979. A possible sensory function for the stop-go patterns of running in phorid flies. Physiol. Entomol. 4, 361-370.

Morgan, T.H., 1926. Genetics and the physiology of development. Am. Nat. LX 1, 489-515.

Neckameyer, W., 1996. Multiple roles for dopamine in Drosophila development. Dev. Biol. 176, 209-219.

Neckameyer, W.S., Cooper, R.L., 1998. GABA transporters in Drosophila melanogaster: developmental expression, behavior, and physiology. Invert. Neurosci. 3, 279-294.

Petersen, S.A., Fetter, R.D., Noordemeer, J.N., Goodman, C.S., DiAntonio, A., 1997. Genetic analysis of glutamate receptors in Drosophila reveals a retrograde signal regulating presynaptic transmitter release. Neuron 18, 1237-1248.

Plunkett, C.R., 1926. The interaction of genetic and environmental factors in development. J. Exp. Zool. 46, 181-244.

Sandrock, A.W., Dryer, S.E., Rosen, K.M., Gozani, S.N., Kramer, R., Theill, L.E., et al., 1997. Maintenance of acetylcholine receptor number by neuregulin at the neuromuscular junction in vivo. Science 276, 599-604.

Sewell, D., Burnet, B., Connolly, K., 1975. Genetic analysis of larval feeding behavior in Drosophila melanogaster. Genet. Res. Cambridge 24, 163-173.

Stewart, B.A., Atwood, H.L., Renger, J.J., Wang, J., Wu, C.F., 1994. Improved stability of Drosophila larval neuromuscular preparation in haemolymph-like physiological solutions. J. Comp. Physiol. A 175, 179-191.

Stewart, B.A., Schuster, C.M., Goodman, C.S., Atwood, H.L., 1996. Homeostasis of synaptic transmission in Drosophila with genetically altered nerve terminal morphology. J. Neurosci. 16, 3877-3886.

Traut, W., 1994. Sex determination in the fly Megaselia scalaris, a model system for primary steps of sex chromosome evolution. Genetics 136, 1097-1104.

Willhoeft, U., Traut, W., 1990. Molecular differentiation of the homomorphic sex chromosomes in Megaselia scalaris (Diptera) detected by random DNA probes. Chromosoma 99, 237-242.

Wolf, K.W., Jeppesen, P., Mitchell, A., 1993. Spermatid nucleus of Megaselia scaris Loew (Insecta, Diptera, Phoridae): a study using anti-histone antibodies, scanning electron microscopy, and a centromere-specific oligonucleotide. Mol. Reprod. Dev. 35, 272-276. 